EISSN: 2706-7955 ISSN: 2077-4605

DOI: 10.36632/mejar/2021.10.3.63

Journal homepage: www.curresweb.com

Pages: 964-981

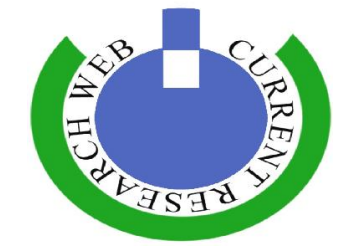

\title{
A Model for Study of Some Soils Properties Adjacent to El Kharga - Baris Road, Egypt
}

\section{Moamen M. El Kady}

Department of Pedology, Desert Research Centre, El-Matariya 11753, Cairo, Egypt

Received: 10 July $2021 \quad$ Accepted: 30 August $2021 \quad$ Published: 10 Sept. 2021

\begin{abstract}
Egypt targets to increase agricultural production by $30 \%$ in 2024 and, that will lead to enhancement of income of small farmers, whilst increasing exports. Hence, the Egyptian government is constantly striving to find out new land resources for agriculture. The study area, covering an area of 1000 fed., was chosen as a model for investigating and evaluating some soils adjacent to the west of El Kharga Baris road, New Valley Governorate. Also, for generalizing results of the study to other soils that have the same pedological characteristics. Therefore, this paper aimed to (i) understand soil properties, (ii) characterize of soil types and their spatial distribution based on a semi-detailed grid sampling technique, and (iii) appraise of the investigated land resources for agriculture. The research area is situated between $30^{\circ} 32^{\prime} 35.4$ to $30^{\circ} 33^{\prime} 45.3^{\prime \prime} \mathrm{E}$ and $25^{\circ} 00^{\prime} 27.4^{\prime \prime}$ to $25^{\circ} 01^{\prime} 49.3^{\prime \prime} \mathrm{N}$. The soils are could be divided to, (1) deep coarse-textured soils which covered $2.89 \%$ of the studied area, (2) deep moderately-fine textured soils with a sandy surface; $47.38 \%$, (3) deep to moderately deep moderately-fine textured soils with gypsum hardpan and sandy surface; $39.77 \%$, and (4) shallow coarse-textured soils; $6 \%$. The soils could be evaluated based on capability into classes II, III, and VI represented 3.55, 86.58, and 6\%, respectively. The results referred that, $90.13 \%$ of the studied area is suitable for agriculture with moderate to special conservation practices. Thus, the proposed practices are concern with in establishing featured systems of subsurface drainage and irrigation that can alleviate the constraints of agricultural production and enhance the prolificacy.
\end{abstract}

Keywords: Land capability, Spatial distribution, Soil properties, Evaluation for agriculture, El Kharga, Egypt

\section{Introduction}

Pedological investigations and explorations are conducted to get clear information about the soil properties for agricultural landuses and their proper management. In addition to, detailed or semidetailed soil studies are urgent for precision agriculture which is necessary for improving resource use efficiency, quality, profitability and sustainability of agricultural production.

Brady and Weil (2016) stated that soil surveys are most worthy if they characterize and delineate natural soil bodies which in turn can assist to creating interpretations for all types of soil uses. Also, using of recent techniques like spatial soil information systems are playing a more and more important role in this context (Lagacherie and McBratney, 2004; Rossiter, 2004).

One of agricultural strategies which are undertaken by Egyptian government is maximizing the exploitation of natural resources for agriculture output so as to facing the urgent demand for food. New Valley governorate is one of the important portions for agricultural expansion where it covers about 44 $\%$ of the total surface area of Egypt and occupying the southern half of its western desert, (Gameh et al., 2017).

Therefore, about $127.5 \mathrm{Km}^{2}$ of El Kharga depression $\left(\approx 11979.7 \mathrm{Km}^{2}\right)$, were recently selected by the government for conducting soil investigations and evaluate them for agricultural landuse. Gameh et al., (2017) found that the area which they selected for study in El Kharga depression was filled with marine shale sediments covered with sand sheets. 
Previous studies on El Kharga depression were mostly regional and indicated that $62 \%$ of its soils are suitable for agriculture however they need to moderate-high conservation practices. The rest of soils (38\%) are not suitable for cultivation which represented in sand dunes and rock lands, (Gad, 2015).

In the area between $25^{\circ} 30^{\prime}$ and $25^{\circ} 31^{\prime} \mathrm{N}$ and longitudes $30^{\circ} 35^{\prime}$ and $30^{\circ} 36^{\prime} \mathrm{E}$ at El Kharga depression, moderately and marginally suitable soils for agriculture could be improved for agriculture production due to following the proper conservation practices. Where, the most dominant limitations within the area were represented in high soil salinity, alkalinity and sandy texture. It could be recommended that correcting those soil obstacles will improve the land capability much more (Gameh et al., 2017).

The Egyptian government chose the study area as a model (1000 fed.) to evaluate its soils for agriculture by date palms within the national project for planting 2.5 million palm trees in the New Valley Governorate. These palm trees will be exploited to produce the best types of dates for export, where they will provide a hard currency for improving the Egyptian economy.

Therefore, this work has undertaken for; (i) understanding of soil properties related to the study area as a model (1000 fed.), (ii) characterization of soil types and their spatial distribution on basis of a semi-detailed grid sampling technique, and (iii) appraisement of the investigated land resources for agriculture.

\section{Materials and Methods}

\section{Site description}

The study area covers 1000 feddans, adjacent to the west of El Kharga - Baris road, western desert, Egypt between longitudes $30^{\circ} 32^{\prime} 35.4$ to $30^{\circ} 33^{\prime} 45.3^{\prime \prime} \mathrm{E}$ and latitudes $25^{\circ} 00^{\prime} 27.4^{\prime \prime}$ to $25^{\circ} 01^{\prime} 49.3^{\prime \prime} \mathrm{N}$ as represented in Fig. (1). Kharga Oasis is characterized by tropical arid climate. The maximum day time temperature fluctuates within a wide range, reaching up to $45-50{ }^{\circ} \mathrm{C}$ in summer months, meanwhile in winter, the minimum temperature may drop to as low as zero at night. Wind speed tends to be low in August and reaches a peak from March to May causing dust storms famously known as "El-Khamasin". The annual mean value of relative humidity is about $39 \%$. Rainfall is extremely scarce and insignificant $(\sim 1 \mathrm{~mm} /$ year $)$, occasional heavy rainstorms severely attack the area from time to time (Salman et al., 2010).

Geologically, the Lower Cretaceous-Lower Tertiary sedimentary sequence overlies unconformable the Precambrian basement rocks at Kharga basin. This sedimentary sequence comprises the Nubian sandstone overlain by variegated shale rock units, which are well exposed forming most bedrocks of the depression floor. These widely exposed rock units are followed upward by the Duwi, Dakhla, Tarawan, Esna and Thebes formations exposed on the eastern and the northern scarps bounding the depression. Furthermore, the Quaternary times in the study area were characterized by alternating periods of wet and dry climates, which resulted in several fluvial, lacustrine and aeolian deposits strewn on the depression floor (Beadnell 1933, El-Sankary 2002, Salman et al., 2010), Fig. (2).

Geomorphologically, the landscape of El Kharga depression is considered as either high plateau in the northern and eastern boundaries, or low-lying depression floor where the studied area is located, meanwhile the pediment areas in-between, are considered as badlands, (Salman et al., 2010).

\section{Soil sampling and laboratory analyses}

On basis of a semi-detailed grid sampling technique, eighteen soil profiles represented the investigation area were digged and morphologically described applying Soil Survey Manual (Soil Survey Staff, 2017), Figs. (3). Total sixty soil samples were collected from the genetic horizons for physical and chemical analyses. 


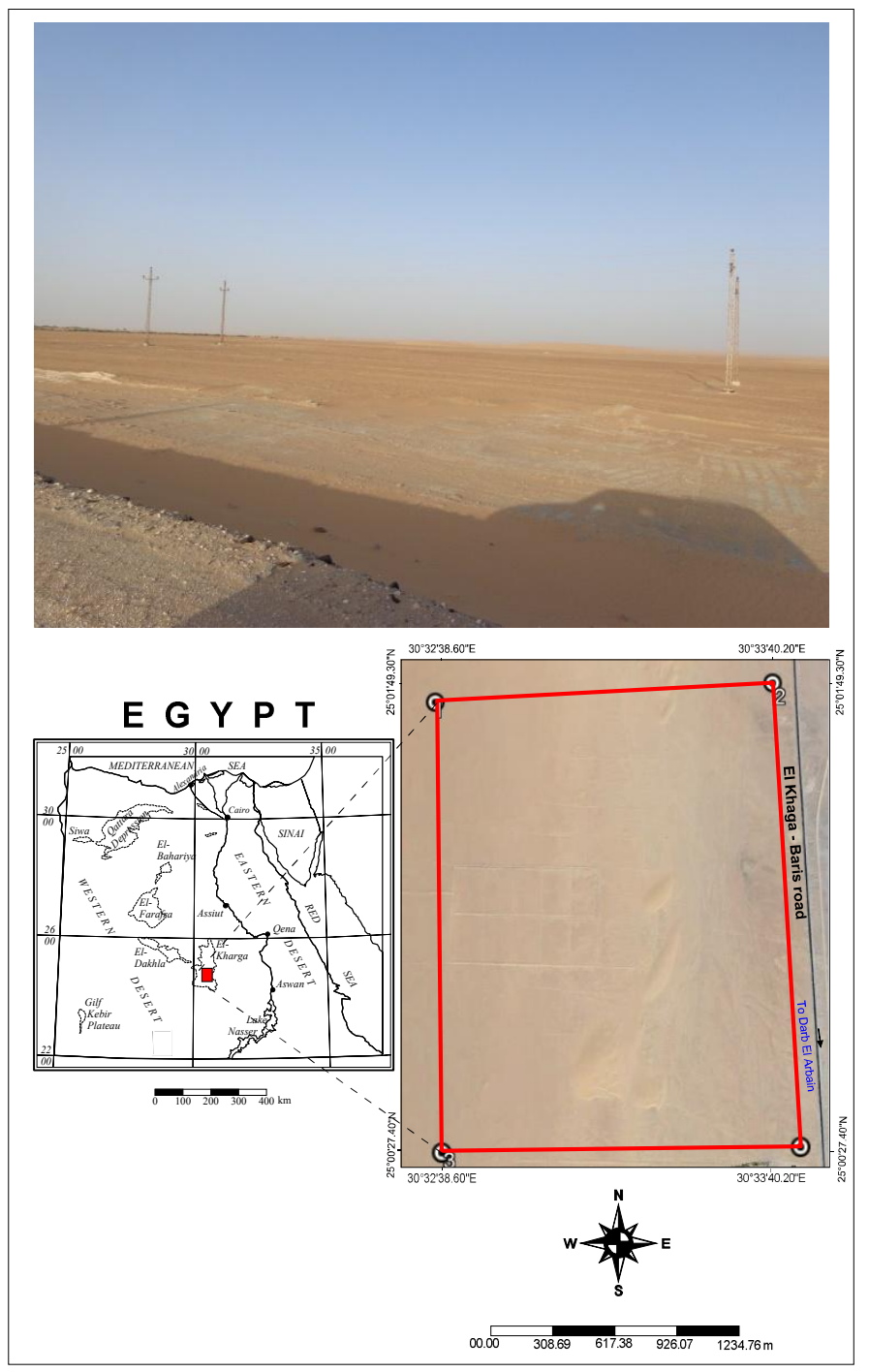

Fig. 1: Location of the studied area

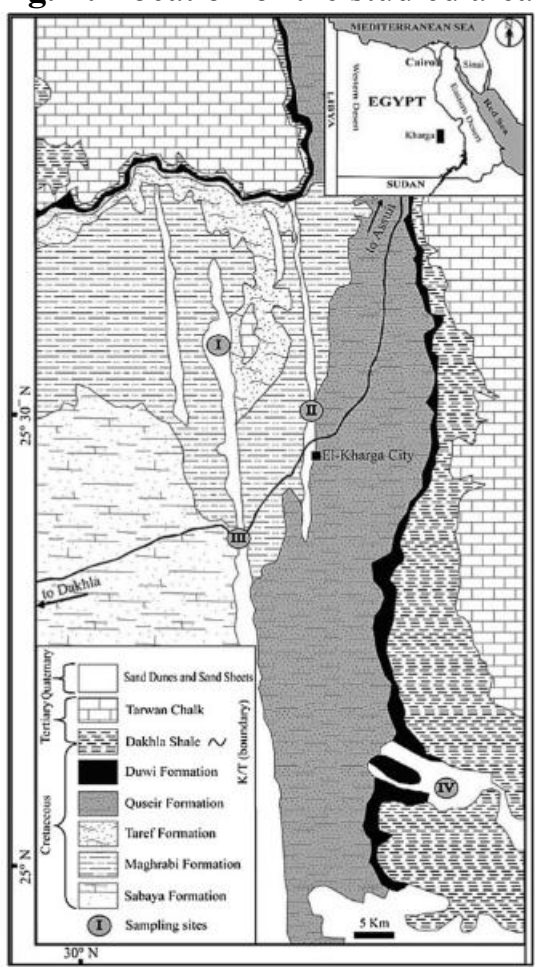

Fig. 2: Geology of El Kharga depression (After CONOCO, 1987 and Abu Seif 2013) 

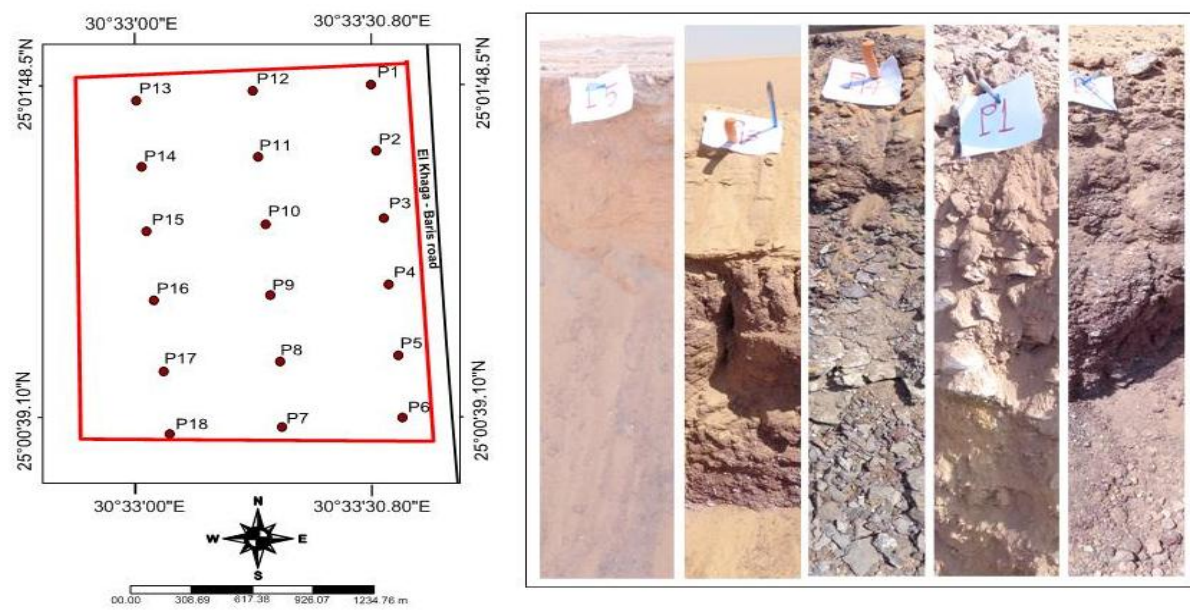

Fig. 3: Soil profiles locations along with a photo for some of them within the studied area

Soil laboratory analyses performed according to (Page et al. 1982) including soil texture, total calcium carbonates, electrical conductivity of soil extracts (EC), and soil reaction (pH). Gypsum was estimated following the method described by (Omran 2016).

\section{Characterization and mapping of Soil types using GIS processing}

A digital database for the site under investigation was generated using Arc-GIS 9.2 (ESRI, 2006) software. Sites of soil observations were digitized, as a unique identifier for each location connected to correlated attribute data using the Database Management System (DBMS). Soil mapping units were produced by overlapping the most variable soil data where final soil and its assessment maps were generated.

\section{Land capability evaluation and statistical analysis}

Land capability classes were defined according to the rating of soil factors adapted by USDA System of land capability classification (Klingebiel and Montgomery, 1961) as shown in Table (1). Data were subjected to a statistical analysis that was conducted by SPSS 21.1 software program (SPSS. 2014).

Table 1: Major soil factors that affect land capability

\begin{tabular}{lll}
\hline Soil Factor & Description & Best Land Class \\
\hline \multirow{3}{*}{ Texture (1): } & Coarse textured & III \\
& Moderately coarse, medium & I \\
& Moderately fine and fine & I \\
\hline \multirow{3}{*}{ Depth (2): } & Deep or moderately deep & I \\
& Shallow & III \\
& Very shallow & VII \\
\hline \multirow{3}{*}{ Slope (3): } & Nearly level (0 to $1 \%)$ & I \\
& Gently sloping (1\% to 3\%) & II \\
& Moderately sloping (3\% to 5\%) & III \\
& Strongly sloping (5\% to $8 \%)$ & IV \\
& Steep and very steep (8\% to $15 \%+)$ & VI \\
\hline \multirow{3}{*}{ Erosion (4): } & None to slight erosion & I \\
& Moderate & II \\
\hline \multirow{3}{*}{ Permeability (5): } & Severe or very severe & VI \\
& Rapid & III \\
& Vederate and slow & I \\
Runoff (6): & Rapid & II \\
& Moderate and slow & III \\
& Very slow & I \\
\hline
\end{tabular}




\section{Results and Discussion}

\section{Soil Morphological Properties}

The surface of the studied area is generally characterized by almost flat topography and nearly level slope not exceeded $2 \%$ and covered by sand sheet with featured ripple marks, and occasional slightly elevated sand dunes. Natural vegetation was scarce and the soil is not cultivated till the time of investigation. Soil depth ranged between deep to moderately deep. The soils were either coarse textured or moderately fine-textured with sandy surface. The natural drainage condition of the soils under profiles 5, 9, 10,11,12, 13,15,16, and 17 classified as well drainer. Where, no phenomena were observed that would impede the root penetration into the soil or reduce its permeability to water.

However, soils of profiles $1,2,3,7,8,14$, and 18 were either poorly or imperfectly drained due to; (i) the spread of gypsic hardpan (Profile 1; $40 \mathrm{~cm}$ ), and (ii) appearance of extremely hard layers of shale and platy-structured clay texture, gypsum, salts, and iron oxides in deep layers of the other profiles. Soil consistency was slightly hard or soft in most of soil horizons, while the deep layer of soil profiles $(11,14,15,16,17$, and 18) sometimes showed a hard nature where they are not represent an obstacle to tree plant growth. Deep soil horizons of profiles 1, 2, 4, and 8 displayed a very hard consistency which would obstruct the locomotion of roots and water in the soil.

Soil structure of surface layers was often weak, where it displayed massive and single grain. While, it showed sub-angular or angular blocky and platy with moderately fine textured sub-surface horizons. Soil colour closely reflected the parent materials and revealed variations of shadows either vertical with depth or horizontally from place to another. Many gypsum formations of various sizes and shapes were clearly present in the form of veins, spots or segregations. Additionally, there were accumulations of salts either in the surface horizons or in the subsurface layers, which corresponds to the formation of a gypsum-saline horizon in profiles of 12, 13, and 17. Also, it was noticed a consolidation by iron oxides in most of the subsurface layers of the soil, Table (2).

\section{Soil Physical and Chemical Properties}

Soil Physicochemical data in table (3) showed high variability within the research site. Total sand ranged between $71 \%$ at surface soils of profile (1) and $99.86 \%$ at the deepest layer of profile (10). The maximum content of silt (19.40\%) was observed at surface horizon of profile (1) while the highest amount of clay (21.67\%) was found at subsurface layer of profile (3). Accordingly, particle size distribution results referred that soil texture class fell between medium sand to sandy clay loam. Gravel content was not exceeded $12.35 \%$, however most of soil samples were free of rock fragments. The soils were slightly to very strongly alkaline with $\mathrm{pH}$ ranged from 7.40 to 9.13 . The electrical conductivity (EC) varied between 2.07 to $180.00 \mathrm{dS} \mathrm{m}^{-1}$ indicating that the soil diversified between free from salts to strongly saline. Calcium carbonate content assorted between non-effervescence to strongly effervescence with $\mathrm{HCl}$, where it was not overrun $9.47 \%$ within the studied profiles. Gypsum amounts ranged between naught or traces to $12.58 \%$.

\section{Characterization of Soil Types and Their Spatial Variability}

On basis of soil morphology, physical and chemical properties of the researched area, four different soil mapping units could be distinguished in the following; (i) deep coarse textured soils which represented $2.89 \%$ of the studied area; SMU1, (ii) deep moderately-fine textured soils with sandy surface, covered an area of $47.38 \%$; SMU2, (iii) deep to moderately deep moderately-fine textured soils with gypsum hardpan and sandy surface which comprised an area of 39.77\%; SMU3, and (iv) shallow coarse textured soils that included an area of $6 \%$; SMU4. The sand dunes covered an area of $3.96 \%$ of the total, Fig. (4). 
Table 2: Main morphological characteristics of the investigated soils

\begin{tabular}{|c|c|c|c|c|c|c|c|c|c|c|c|}
\hline \multirow{2}{*}{$\begin{array}{l}\text { Depth } \\
\text { (cm) }\end{array}$} & \multirow{2}{*}{ Colour } & \multirow{2}{*}{$\begin{array}{l}\text { Texture } \\
\text { (USDA) }\end{array}$} & \multirow{2}{*}{$\begin{array}{c}\text { Coarse } \\
\text { Fragments }\end{array}$} & \multirow{2}{*}{ Structure } & \multicolumn{2}{|c|}{ Consistence } & \multirow{2}{*}{$\begin{array}{c}\text { Gypsum } \\
\text { Formation }\end{array}$} & \multirow{2}{*}{$\begin{array}{l}\text { Redoximorphic } \\
\text { Features } \\
(\mathrm{Fe} \text { and/or } \mathrm{Mn})^{2}\end{array}$} & \multirow{2}{*}{ Roots } & \multirow{2}{*}{$\begin{array}{c}\text { Reaction } \\
\text { with } \\
\text { HCl }\end{array}$} & \multirow{2}{*}{ Boundary } \\
\hline & & & & & Dry & Wet & & & & & \\
\hline \multicolumn{12}{|c|}{ Soil Profile (1) } \\
\hline 40 & \multicolumn{11}{|c|}{ G Y P S U M H A R D P A N } \\
\hline \multicolumn{12}{|c|}{ Soil Profile (2) } \\
\hline 0-10 & $7.5 \mathrm{YR} 6 / 4$ & fs & - & $\mathrm{m}$ & $\mathrm{s}$ & so, po & - & - & - & es & as \\
\hline $60-90$ & 7.5YR $6 / 4$ & $\mathrm{scl}$ & - & sbk & vh & $\mathrm{s}, \mathrm{p}$ & $\mathrm{cf}$ & - & - & es & as \\
\hline \multicolumn{12}{|c|}{ Soil Profile (3) } \\
\hline $0-10$ & 7.5YR 6/4 & fs & - & sg & 1 & so, po & - & - & - & es & as \\
\hline $10-60$ & $7.5 \mathrm{YR} 7 / 2$ & $\mathrm{scl}$ & $\mathrm{ffg}$ & sbk & sh & $\mathrm{s}, \mathrm{p}$ & $\mathrm{cf}-\mathrm{m}$ & $\mathrm{cf}-\mathrm{m}$ & - & $\mathrm{e}$ & as \\
\hline $60-90$ & $7.5 \mathrm{YR} 6 / 2$ & $\mathrm{scl}$ & - & abk & $\mathrm{h}$ & $\mathrm{s}, \mathrm{p}$ & $\mathrm{cf}-\mathrm{m}$ & $\mathrm{cf}-\mathrm{m}$ & - & - & as \\
\hline \multicolumn{12}{|c|}{ Soil Profile (4) } \\
\hline 0-10 & $7.5 Y R 6 / 4$ & fs & - & $\mathrm{sg}$ & 1 & so, po & - & - & - & e & as \\
\hline $60-90$ & 10 YR $5 / 2$ & $\mathrm{scl}$ & - & abk & $\mathrm{h}$ & $\mathrm{s}, \mathrm{p}$ & $\mathrm{mf}-\mathrm{m}$ & $\mathrm{cf}-\mathrm{m}$ & - & $\mathrm{e}$ & as \\
\hline $90-150$ & 10YR $6 / 2$ & $\mathrm{scl}$ & - & abk & $\mathrm{vh}$ & $\mathrm{s}, \mathrm{p}$ & $\mathrm{mf}-\mathrm{m}$ & & - & - & - \\
\hline \multicolumn{12}{|c|}{ Soil Profile (5) } \\
\hline $0-15$ & $7.5 Y R 7 / 6$ & fs & - & $\mathrm{m}$ & $\mathrm{s}$ & so, po & - & - & - & e & cs \\
\hline $15-50$ & 7.5YR 7/6 & $\mathrm{ms}$ & ffg & $\mathrm{m}$ & $\mathrm{s}$ & so, po & - & - & - & e & ds \\
\hline $50-100$ & 7.5YR 7/6 & $\mathrm{ms}$ & - & $\mathrm{m}$ & $\mathrm{s}$ & so, po & - & - & - & e & ds \\
\hline 100-150 & 7.5YR 7/6 & $\mathrm{ms}$ & - & $\mathrm{m}$ & $\mathrm{s}$ & so, po & - & - & - & $\mathrm{e}$ & - \\
\hline
\end{tabular}




\section{Continue,}

\begin{tabular}{|c|c|c|c|c|c|c|c|c|c|c|c|}
\hline \multirow{2}{*}{ Depth (cm) } & \multirow{2}{*}{ Colour } & \multirow{2}{*}{ Texture (USDA) } & \multirow{2}{*}{$\begin{array}{c}\text { Coarse } \\
\text { Fragments }\end{array}$} & \multirow{2}{*}{ Structure } & \multicolumn{2}{|c|}{ Consistence } & \multirow{2}{*}{$\begin{array}{c}\text { Gypsum } \\
\text { Formation }^{1}\end{array}$} & \multirow{2}{*}{$\begin{array}{l}\text { Redoximorphic Features } \\
\quad(\mathrm{Fe} \text { and/or } \mathrm{Mn})^{2}\end{array}$} & \multirow{2}{*}{ Roots } & \multirow{2}{*}{ Reaction with $\mathrm{HCl}$} & \multirow{2}{*}{ Boundary } \\
\hline & & & & & Dry & Wet & & & & & \\
\hline \multicolumn{12}{|c|}{ Soil Profile (6) } \\
\hline $0-20$ & 7.5 YR $6 / 4$ & vfs & - & sg & 1 & so, po & - & - & - & es & as \\
\hline $20-60$ & 7.5 YR $6 / 4$ & $\mathrm{scl}$ & $\mathrm{ffg}$ & sbk & sh & $\mathrm{s}, \mathrm{p}$ & $\mathrm{cf}-\mathrm{m}$ & cf-m & - & $\mathrm{e}$ & cs \\
\hline $60-90$ & 10YR 6/2 & $\mathrm{scl}$ & - & sbk & sh & $\mathrm{s}, \mathrm{p}$ & - & - & - & $\mathrm{e}$ & cs \\
\hline 90-150 & 7.5 YR $6 / 2$ & $\mathrm{scl}$ & - & sbk & sh & $\mathrm{s}, \mathrm{p}$ & - & - & - & $\mathrm{e}$ & - \\
\hline \multicolumn{12}{|c|}{ Soil Profile (7) } \\
\hline $0-50$ & 7.5 YR $7 / 6$ & fs & - & sg & 1 & so, po & - & - & - & es & as \\
\hline $50-100$ & $5 \mathrm{YR} 5 / 3$ & $\mathrm{scl}$ & - & $\mathrm{pl}$ & sh & $\mathrm{s}, \mathrm{p}$ & - & $\mathrm{cf}-\mathrm{m}$ & - & $\mathrm{e}$ & $\mathrm{cs}$ \\
\hline $100-130$ & 10YR $6 / 2$ & $\mathrm{scl}$ & - & $\mathrm{pl}$ & sh & $\mathrm{s}, \mathrm{p}$ & - & $\mathrm{cf}-\mathrm{m}$ & - & $\mathrm{e}$ & as \\
\hline \multicolumn{12}{|c|}{ Soil Profile (8) } \\
\hline $0-10$ & $5 Y R 6 / 3$ & fs & - & $\mathrm{m}$ & $\mathrm{s}$ & so, po & - & - & - & es & as \\
\hline $10-30$ & $5 Y R 5 / 3$ & $\mathrm{scl}$ & $\mathrm{ffg}$ & sbk & $\operatorname{sh}$ & $\mathrm{s}, \mathrm{p}$ & $\mathrm{mf}$ & - & - & $\mathrm{e}$ & cs \\
\hline $30-90$ & $5 \mathrm{YR} 5 / 3$ & $\mathrm{scl}$ & - & sbk & $\mathrm{sh}$ & $\mathrm{s}, \mathrm{p}$ & $\mathrm{cf}-\mathrm{m}$ & - & - & $\mathrm{e}$ & as \\
\hline 90-130 & 10YR 6/4 & $\mathrm{scl}$ & - & $\mathrm{pl}$ & vh & $\mathrm{s}, \mathrm{p}$ & $\mathrm{ff}$ & cf-m & - & $\mathrm{e}$ & as \\
\hline \multicolumn{12}{|c|}{ Soil Profile (9) } \\
\hline $0-50$ & 7.5YR 7/6 & fs & - & $\mathrm{m}$ & $\mathrm{s}$ & so, po & - & - & - & es & as \\
\hline $50-100$ & $7.5 \mathrm{YR} 6 / 4$ & $\mathrm{scl}$ & - & sbk & $\mathrm{sh}$ & $\mathrm{s}, \mathrm{p}$ & - & - & - & es & as \\
\hline $100-150$ & 5 YR 5/3 & $\mathrm{scl}$ & - & sbk & $\mathrm{h}$ & $\mathrm{s}, \mathrm{p}$ & $\mathrm{cvf}$ & $\mathrm{cf}-\mathrm{m}$ & - & es & - \\
\hline \multicolumn{12}{|c|}{ Soil Profile (10) } \\
\hline $0-20$ & $7.5 \mathrm{YR} 6 / 4$ & $\mathrm{scl}$ & - & $\mathrm{pl}$ & $\mathrm{sh}$ & $\mathrm{s}, \mathrm{p}$ & - & - & - & es & as \\
\hline $20-70$ & 10YR 7/6 & $\mathrm{scl}$ & - & sbk & $\mathrm{sh}$ & $\mathrm{s}, \mathrm{p}$ & fvf & - & - & $\mathrm{e}$ & as \\
\hline $70-90$ & 7.5YR 6/4 & $\mathrm{scl}$ & $\mathrm{ffg}$ & $\mathrm{m}$ & $\mathrm{sh}$ & $\mathrm{s}, \mathrm{p}$ & fvf & - & - & $\mathrm{e}$ & as \\
\hline 90-150 & $7.5 \mathrm{YR} 7 / 6$ & $\mathrm{~ms}$ & $\mathrm{ffg}$ & $\mathrm{m}$ & $\mathrm{s}$ & so, po & - & - & - & $\mathrm{e}$ & - \\
\hline
\end{tabular}




\section{Continue,}

\begin{tabular}{|c|c|c|c|c|c|c|c|c|c|c|c|}
\hline \multirow{2}{*}{ Depth (cm) } & \multirow{2}{*}{ Colour } & \multirow{2}{*}{ Texture (USDA) } & \multirow{2}{*}{$\begin{array}{l}\text { Coarse } \\
\text { Fragments }\end{array}$} & \multirow{2}{*}{ Structure } & \multicolumn{2}{|c|}{ Consistence } & \multirow{2}{*}{$\begin{array}{l}\text { Gypsum } \\
\text { Formation }{ }^{1}\end{array}$} & \multirow{2}{*}{$\begin{array}{l}\text { Redoximorphic } \\
\text { Features } \\
(\text { Fe and/or Mn })^{2}\end{array}$} & \multirow{2}{*}{ Roots } & \multirow{2}{*}{ Reaction with $\mathrm{HCl}$} & \multirow{2}{*}{ Boundary } \\
\hline & & & & & Dry & Wet & & & & & \\
\hline \multicolumn{12}{|c|}{ Soil Profile (11) } \\
\hline $0-10$ & 7.5YR 6/4 & fs & - & $\mathrm{sg}$ & 1 & so, po & - & - & - & es & as \\
\hline $10-60$ & 7.5YR 6/6 & $\mathrm{scl}$ & ffg & sbk & $\mathrm{sh}$ & $\mathrm{s}, \mathrm{p}$ & fvf & - & - & es & as \\
\hline $60-90$ & 7.5YR 6/6 & fs & - & $\mathrm{m}$ & $\mathrm{sh}$ & so, po & - & - & - & es & as \\
\hline $90-150$ & $5 Y R 6 / 4$ & $\mathrm{scl}$ & - & sbk & $\mathrm{h}$ & $\mathrm{s}, \mathrm{p}$ & $\mathrm{cf}-\mathrm{m}$ & - & - & es & - \\
\hline \multicolumn{12}{|c|}{ Soil Profile (12) } \\
\hline $0-50$ & $10 Y R 7 / 6$ & fs & - & $\mathrm{sg}$ & 1 & so, po & - & - & - & es & gs \\
\hline $50-100$ & 7.5YR 7/6 & $\mathrm{ms}$ & - & sg & 1 & so, po & - & - & - & $\mathrm{e}$ & ds \\
\hline $100-150$ & 7.5YR 7/6 & fs & - & $\mathrm{sg}$ & 1 & so, po & - & - & - & es & - \\
\hline \multicolumn{12}{|c|}{ Soil Profile (13) } \\
\hline $0-15$ & $10 Y R 7 / 6$ & fs & - & $\mathrm{m}$ & $\mathrm{s}$ & so, po & - & - & - & es & as \\
\hline $15-60$ & $5 Y R 6 / 3$ & $\mathrm{scl}$ & ffg & cpr & $\mathrm{sh}$ & $\mathrm{s}, \mathrm{p}$ & - & - & - & $\mathrm{e}$ & as \\
\hline $60-150$ & 2.5 YR $6 / 2$ & $\mathrm{scl}$ & - & sbk & sh & $\mathrm{s}, \mathrm{p}$ & $\mathrm{cf}-\mathrm{m}-\mathrm{c}$ & - & - & - & - \\
\hline \multicolumn{12}{|c|}{ Soil Profile (14) } \\
\hline $\mathbf{0 - 3 0}$ & $5 Y R 6 / 3$ & $\mathrm{scl}$ & - & $\mathrm{m}$ & $\mathrm{s}$ & $\mathrm{s}, \mathrm{p}$ & - & - & - & e & as \\
\hline $30-70$ & $5 \mathrm{YR} 5 / 2$ & $\mathrm{scl}$ & ffg & $\mathrm{pl}$ & sh & $\mathrm{s}, \mathrm{p}$ & cf-m & $\mathrm{cf}-\mathrm{m}$ & - & $\mathrm{e}$ & as \\
\hline 70-100 & $10 \mathrm{YR} 8 / 2$ & $\mathrm{scl}$ & - & abk & $\mathrm{h}$ & $\mathrm{s}, \mathrm{p}$ & cf-m & cf-m & - & - & as \\
\hline \multicolumn{12}{|c|}{ Soil Profile (15) } \\
\hline $0-15$ & 7.5YR 7/6 & fs & - & $\mathrm{m}$ & $\mathrm{s}$ & so, po & - & - & - & es & as \\
\hline $15-70$ & $2.5 \mathrm{YR} 5 / 4$ & $\mathrm{scl}$ & - & cpr & sh & $\mathrm{s}, \mathrm{p}$ & - & - & - & e & as \\
\hline $70-150$ & $5 Y R 5 / 3$ & $\mathrm{scl}$ & - & sbk & $\mathrm{h}$ & $\mathrm{s}, \mathrm{p}$ & cf-m & - & - & e & - \\
\hline
\end{tabular}




\section{Continue,}

\begin{tabular}{|c|c|c|c|c|c|c|c|c|c|c|c|}
\hline \multirow[b]{2}{*}{ Depth (cm) } & \multirow[b]{2}{*}{ Colour } & \multirow[b]{2}{*}{ Texture (USDA) } & \multirow{2}{*}{$\begin{array}{c}\text { Coarse } \\
\text { Fragments }\end{array}$} & \multirow[b]{2}{*}{ Structure } & \multicolumn{2}{|c|}{ Consistence } & \multirow{2}{*}{$\begin{array}{c}\text { Gypsum } \\
\text { Formation }^{1}\end{array}$} & \multirow{2}{*}{$\begin{array}{c}\text { Redoximorphic } \\
\text { Features } \\
\text { (Fe and/or } \\
\text { Mn })^{2}\end{array}$} & \multirow[b]{2}{*}{ Roots } & \multirow[b]{2}{*}{ Reaction with $\mathrm{HCl}$} & \multirow[b]{2}{*}{ Boundary } \\
\hline & & & & & Dry & Wet & & & & & \\
\hline \multicolumn{12}{|c|}{ Soil Profile (16) } \\
\hline 0-30 & 7.5YR 7/6 & fs & - & sg & 1 & so, po & - & - & - & e & as \\
\hline $30-80$ & $5 Y R 5 / 2$ & $\mathrm{scl}$ & - & sbk & $\mathrm{sh}$ & $\mathrm{s}, \mathrm{p}$ & - & - & - & e & as \\
\hline $80-150$ & $2.5 \mathrm{YR} 5 / 2$ & $\mathrm{scl}$ & - & sbk & $\mathrm{h}$ & $\mathrm{s}, \mathrm{p}$ & $\mathrm{cf}-\mathrm{m}$ & - & - & $\mathrm{e}$ & - \\
\hline \multicolumn{12}{|c|}{ Soil Profile (17) } \\
\hline 0-10 & 7.5YR 7/6 & fs & - & sg & 1 & so, po & - & - & - & es & as \\
\hline $10-30$ & 7.5YR 6/4 & $\mathrm{scl}$ & $\mathrm{ffg}$ & cpr & $\mathrm{s}$ & $\mathrm{s}, \mathrm{p}$ & - & - & - & es & as \\
\hline $30-80$ & 7.5YR $6 / 4$ & $\mathrm{scl}$ & - & sbk & sh & $\mathrm{s}, \mathrm{p}$ & $\mathrm{ff}$ & - & - & $\mathrm{e}$ & as \\
\hline $80-150$ & 7.5YR 5/4 & $\mathrm{scl}$ & - & sbk & $\mathrm{h}$ & $\mathrm{s}, \mathrm{p}$ & $\mathrm{cf}-\mathrm{m}$ & - & - & $\mathrm{e}$ & - \\
\hline \multicolumn{12}{|c|}{ Soil Profile (18) } \\
\hline 0-30 & 10YR 7/6 & fs & ffg & sg & 1 & so, po & - & - & - & es & as \\
\hline $30-60$ & 7.5YR 6/4 & $\mathrm{scl}$ & - & cpr & $\mathrm{sh}$ & $\mathrm{s}, \mathrm{p}$ & $\mathrm{cf}-\mathrm{m}$ & - & - & $\mathrm{e}$ & as \\
\hline $60-100$ & 7.5 YR $6 / 2$ & $\mathrm{scl}$ & - & $\mathrm{pl}$ & $\mathrm{h}$ & $\mathrm{s}, \mathrm{p}$ & $\mathrm{cf}-\mathrm{m}$ & cf-m & - & $\mathrm{e}$ & as \\
\hline
\end{tabular}

\begin{tabular}{ll} 
Abbreviations: & \\
\hline $\begin{array}{l}\text { Texture (USDA): } \\
\text { Coarse }\end{array}$ & ls-loamy sand; scl-sandy clay loam; fs-fine sand; ms-medium sand; vfs-very fine sand \\
\hline Sragments: & ffg-few fine gravel; cfg-common fine gravel \\
\hline Consture: & m-massive; abk-angular blocky; sbk-subangular blocky; sg-single grain; pl-platy; cpr-columnar \\
\hline $\mathbf{1 , 2}$ & s-soft; vh-very hard; h-hard; l-loose; sh-slightly hard; ss-slightly; s-sticky; so-non sticky; ps-slightly plastic; p-plastic; po-non plastic \\
\hline & $\begin{array}{l}\text { cf-m: common fine and medium; mf-many fine; cf-common fine; mf-m: many fine \& medium; ff-few } \\
\text { fine; cvf-common very fine; fvf-few very fine; cf-m-c: common fine, medium and coarse }\end{array}$ \\
\hline & Boundary: as-abrupt smooth; cs-clear smooth; ds-diffuse smooth; gs-gradual smooth \\
\hline
\end{tabular}


Table 3: Some physicochemical characteristics of the investigated soils

\begin{tabular}{|c|c|c|c|c|c|c|c|c|c|c|c|c|c|c|c|}
\hline \multirow{2}{*}{$\begin{array}{l}\text { Depth } \\
\text { (cm) }\end{array}$} & \multirow{2}{*}{$\begin{array}{c}\text { EC } \\
\text { dS } \mathbf{~ m}^{-1}\end{array}$} & \multirow[b]{2}{*}{$\mathbf{p H}$} & \multirow{2}{*}{$\begin{array}{c}\mathrm{CaCO}_{3} \\
\%\end{array}$} & \multirow{2}{*}{$\begin{array}{c}\text { Gypsum } \\
\%\end{array}$} & \multirow{2}{*}{$\begin{array}{c}\text { Gravel } \\
\%\end{array}$} & \multicolumn{9}{|c|}{ Particle Size Distribution } & \multirow{2}{*}{$\begin{array}{l}\text { Texture } \\
\text { (USDA) }\end{array}$} \\
\hline & & & & & & $\begin{array}{c}1-2 \\
(\mathrm{~mm})\end{array}$ & $\begin{array}{l}1-0.5 \\
(\mathrm{~mm})\end{array}$ & $\begin{array}{c}0.5-0.25 \\
(\mathrm{~mm})\end{array}$ & $\begin{array}{c}\text { 0.25-0.125 } \\
(\mathrm{mm})\end{array}$ & $\begin{array}{c}\begin{array}{c}0.125-0.063 \\
(\mathrm{~mm})\end{array} \\
\end{array}$ & $\begin{array}{c}<0.063 \\
(\mathrm{~mm}) \\
\end{array}$ & $\begin{array}{c}\text { Sand } \\
\%\end{array}$ & $\begin{array}{l}\text { Silt } \\
\%\end{array}$ & $\begin{array}{c}\text { Clay } \\
\%\end{array}$ & \\
\hline \multicolumn{16}{|c|}{ Soil Profile (1) } \\
\hline $0-40$ & 5.33 & 8.62 & 1.67 & - & - & - & - & - & - & - & - & 71.00 & 19.40 & 9.60 & ls \\
\hline 40 & & & & & & & & P S U M & H A R D P A & & & & & & \\
\hline \multicolumn{16}{|c|}{ Soil Profile (2) } \\
\hline 0-10 & 27.81 & 8.62 & 6.69 & $\operatorname{tr}^{1}$ & - & 5.40 & 7.73 & 17.46 & 29.95 & 27.67 & 11.79 & - & - & - & fs \\
\hline $10-60$ & 116.00 & 8.66 & 6.41 & 11.02 & 8.89 & - & - & - & - & - & - & 75.50 & 3.00 & 21.50 & $\mathrm{scl}$ \\
\hline $60-90$ & 79.00 & 8.82 & 6.41 & 9.59 & - & - & - & - & - & - & - & 78.00 & 2.00 & 20.00 & $\mathrm{scl}$ \\
\hline \multicolumn{16}{|c|}{ Soil Profile (3) } \\
\hline $0-10$ & 7.71 & 8.53 & 7.17 & - & - & 3.73 & 6.97 & 16.88 & 35.26 & 31.78 & 5.38 & - & - & - & fs \\
\hline $10-60$ & 79.20 & 8.19 & 1.86 & 9.60 & 10.99 & - & - & - & - & - & - & 79.50 & 0.50 & 20.00 & $\mathrm{scl}$ \\
\hline $60-90$ & 13.57 & 8.11 & 0.00 & 7.06 & - & - & - & - & - & - & - & 78.33 & 0.00 & 21.67 & $\mathrm{scl}$ \\
\hline \multicolumn{16}{|c|}{ Soil Profile (4) } \\
\hline $0-10$ & 14.16 & 8.75 & 3.06 & tr. & - & 2.55 & 8.66 & 25.05 & 35.02 & 21.70 & 7.02 & - & - & - & fs \\
\hline $10-60$ & 20.64 & 8.67 & 1.82 & 7.33 & 12.35 & - & - & - & - & - & - & 79.80 & 0.00 & 20.20 & $\mathrm{scl}$ \\
\hline $60-90$ & 156.20 & 7.59 & 0.38 & 12.58 & - & - & - & - & - & - & - & 79.50 & 0.17 & 20.33 & $\mathrm{scl}$ \\
\hline $90-150$ & 126.80 & 7.34 & 0.00 & 11.44 & - & - & - & - & - & - & - & 77.95 & 2.01 & 20.04 & $\mathrm{scl}$ \\
\hline
\end{tabular}




\section{Continue,}

\begin{tabular}{|c|c|c|c|c|c|c|c|c|c|c|c|c|c|c|c|}
\hline \multirow[b]{2}{*}{$\begin{array}{l}\text { Depth } \\
\text { (cm) }\end{array}$} & \multirow[b]{2}{*}{$\begin{array}{c}\text { EC } \\
\text { dS } \mathbf{~ m}^{-1}\end{array}$} & \multirow[b]{2}{*}{ pH } & \multirow[b]{2}{*}{$\begin{array}{c}\mathrm{CaCO}_{3} \\
\%\end{array}$} & \multirow[b]{2}{*}{$\begin{array}{c}\text { Gypsum } \\
\%\end{array}$} & \multirow[b]{2}{*}{$\begin{array}{c}\text { Gravel } \\
\%\end{array}$} & \multicolumn{9}{|c|}{ Particle Size Distribution } & \multirow[b]{2}{*}{ Texture (USDA) ${ }^{2}$} \\
\hline & & & & & & $\begin{array}{c}1-2 \\
(\mathrm{~mm})\end{array}$ & $\begin{array}{r}1-0.5 \\
(\mathrm{~mm}) \\
\end{array}$ & $\begin{array}{c}0.5-0.25 \\
(\mathrm{~mm})\end{array}$ & $\begin{array}{c}\begin{array}{c}0.25-0.125 \\
(\mathrm{~mm})\end{array} \\
\end{array}$ & $\begin{array}{c}\text { 0.125-0.063 } \\
(\mathrm{mm})\end{array}$ & $\begin{array}{c}<0.063 \\
(\mathrm{~mm}) \\
\end{array}$ & $\begin{array}{c}\text { Sand } \\
\% \\
\end{array}$ & $\begin{array}{c}\text { Silt } \\
\%\end{array}$ & $\begin{array}{c}\text { Clay } \\
\% \\
\end{array}$ & \\
\hline \multicolumn{16}{|c|}{ Soil Profile (5) } \\
\hline $0-15$ & 12.30 & 8.77 & 1.82 & $\operatorname{tr}^{1}$ & - & 6.29 & 16.16 & 29.06 & 33.52 & 13.08 & 1.89 & - & - & - & fs \\
\hline $\mathbf{1 5 - 5 0}$ & 11.97 & 9.12 & 1.35 & tr. & 6.33 & 2.67 & 6.67 & 49.71 & 37.65 & 2.84 & 0.46 & - & - & - & $\mathrm{ms}$ \\
\hline $50-100$ & 18.29 & 9.05 & 2.69 & tr. & - & 3.20 & 5.33 & 56.85 & 32.93 & 1.40 & 0.29 & - & - & - & $\mathrm{ms}$ \\
\hline $100-150$ & 19.45 & 9.08 & 3.85 & tr. & - & 4.67 & 6.35 & 44.21 & 41.70 & 2.72 & 0.35 & - & - & - & $\mathrm{ms}$ \\
\hline \multicolumn{16}{|c|}{ Soil Profile (6) } \\
\hline $0-20$ & 29.94 & 8.69 & 6.83 & tr. & - & 8.05 & 5.54 & 12.44 & 31.56 & 32.34 & 10.07 & - & - & - & vfs \\
\hline $20-60$ & 28.51 & 8.46 & 1.63 & 7.64 & 7.04 & - & - & - & - & - & - & 79.01 & 0.49 & 20.50 & $\mathrm{scl}$ \\
\hline $60-90$ & 12.16 & 8.37 & 1.30 & $\operatorname{tr}$ & - & - & - & - & & - & - & 78.99 & 1.01 & 20.00 & $\mathrm{scl}$ \\
\hline $90-150$ & 6.29 & 8.24 & 0.42 & - & - & - & - & - & - & - & - & 78.77 & 1.00 & 20.23 & $\mathrm{scl}$ \\
\hline \multicolumn{16}{|c|}{ Soil Profile (7) } \\
\hline $0-50$ & 5.54 & 8.74 & 6.45 & - & - & 5.40 & 11.47 & 20.44 & 41.30 & 19.80 & 1.59 & - & - & - & fs \\
\hline $50-100$ & 8.26 & 8.16 & 0.37 & - & 1.50 & - & - & - & - & - & - & 79.00 & 0.45 & 20.55 & $\mathrm{scl}$ \\
\hline 100-130 & 8.80 & 8.08 & 0.42 & - & - & - & - & - & - & - & - & 78.56 & 1.00 & 20.44 & scl \\
\hline \multicolumn{16}{|c|}{ Soil Profile (8) } \\
\hline $0-10$ & 10.77 & 8.70 & 5.62 & - & - & 5.43 & 7.31 & 19.45 & 41.06 & 23.93 & 2.82 & - & - & - & fs \\
\hline $10-30$ & 112.20 & 8.59 & 3.16 & 10.88 & 5.49 & - & - & - & - & - & - & 79.49 & 0.00 & 20.51 & $\mathrm{scl}$ \\
\hline $30-90$ & 27.43 & 8.69 & 2.28 & 7.59 & - & - & - & - & - & - & - & 78.99 & 0.00 & 21.01 & $\mathrm{scl}$ \\
\hline $90-130$ & 25.62 & 7.55 & 0.60 & 7.52 & - & - & - & - & - & - & - & 78.01 & 1.09 & 20.90 & $\mathrm{scl}$ \\
\hline
\end{tabular}


Continue,

\begin{tabular}{|c|c|c|c|c|c|c|c|c|c|c|c|c|c|c|c|}
\hline \multirow{2}{*}{$\begin{array}{l}\text { Depth } \\
(\mathrm{cm})\end{array}$} & \multirow{2}{*}{$\begin{array}{c}\mathrm{EC} \\
\mathrm{dS} \mathbf{m}^{-1}\end{array}$} & \multirow[b]{2}{*}{ pH } & \multirow{2}{*}{$\begin{array}{c}\mathrm{CaCO}_{3} \\
\%\end{array}$} & \multirow{2}{*}{$\begin{array}{c}\text { Gypsum } \\
\%\end{array}$} & \multirow{2}{*}{$\begin{array}{c}\text { Gravel } \\
\%\end{array}$} & \multicolumn{9}{|c|}{ Particle Size Distribution } & \multirow{2}{*}{$\begin{array}{l}\text { Texture } \\
\text { (USDA) }^{2}\end{array}$} \\
\hline & & & & & & $\begin{array}{c}1-2 \\
(\mathrm{~mm}) \\
\end{array}$ & $\begin{array}{l}1-0.5 \\
(\mathrm{~mm}) \\
\end{array}$ & $\begin{array}{c}0.5-0.25 \\
(\mathrm{~mm})\end{array}$ & $\begin{array}{c}0.25-0.125 \\
(\mathrm{~mm})\end{array}$ & $\begin{array}{c}0.125-0.063 \\
(\mathrm{~mm})\end{array}$ & $\begin{array}{c}<0.063 \\
(\mathrm{~mm}) \\
\end{array}$ & $\begin{array}{c}\text { Sand } \\
\%\end{array}$ & $\begin{array}{c}\text { Silt } \\
\%\end{array}$ & $\begin{array}{c}\text { Clay } \\
\% \\
\end{array}$ & \\
\hline \multicolumn{16}{|c|}{ Soil Profile (9) } \\
\hline $0-50$ & 3.51 & 9.12 & 8.64 & - & - & 7.49 & 7.74 & 18.42 & 39.07 & 26.03 & 1.25 & - & - & - & fs \\
\hline $50-100$ & 12.07 & 8.56 & 7.06 & $\operatorname{tr}^{1}$ & - & - & - & - & - & - & - & 77.99 & 2.00 & 20.01 & $\mathrm{scl}$ \\
\hline $100-150$ & 29.45 & 7.96 & 5.20 & 7.67 & - & - & - & - & - & - & - & 77.80 & 2.10 & 20.10 & $\mathrm{scl}$ \\
\hline \multicolumn{16}{|c|}{ Soil Profile (10) } \\
\hline 0-20 & 29.14 & 8.81 & 5.71 & tr. & - & - & - & - & - & - & - & 78.80 & 0.55 & 20.65 & $\mathrm{scl}$ \\
\hline 20-70 & 26.03 & 8.90 & 2.55 & 7.54 & 1.47 & - & - & - & - & - & - & 78.00 & 1.78 & 20.22 & scl \\
\hline $70-90$ & 17.10 & 8.05 & 2.14 & 7.19 & 5.26 & - & - & - & - & - & - & 78.66 & 1.00 & 20.34 & $\mathrm{scl}$ \\
\hline 90-150 & 2.07 & 8.61 & 2.14 & - & 3.68 & 0.24 & 13.58 & 65.10 & 19.81 & 1.13 & 0.14 & - & - & - & $\mathrm{ms}$ \\
\hline \multicolumn{16}{|c|}{ Soil Profile (11) } \\
\hline 0-10 & 25.78 & 8.76 & 7.80 & $\operatorname{tr}$ & - & 4.42 & 9.25 & 24.96 & 38.85 & 19.17 & 3.35 & - & - & - & fs \\
\hline $10-60$ & 16.54 & 8.77 & 8.50 & 7.17 & 8.33 & - & - & - & - & - & - & 77.79 & 1.95 & 20.26 & $\mathrm{scl}$ \\
\hline $60-90$ & 7.01 & 8.33 & 5.71 & - & - & 5.06 & 11.97 & 29.91 & 34.16 & 15.16 & 3.74 & - & - & - & fs \\
\hline $90-150$ & 22.33 & 7.94 & 6.04 & 7.40 & - & - & - & - & - & - & - & 79.98 & 0.02 & 20.00 & $\mathrm{scl}$ \\
\hline \multicolumn{16}{|c|}{ Soil Profile (12) } \\
\hline $0-50$ & 5.43 & 9.04 & 7.66 & - & - & 7.33 & 5.76 & 22.64 & 45.42 & 18.08 & 0.77 & - & - & - & fs \\
\hline $50-100$ & 180.00 & 8.85 & 3.85 & tr. & - & 3.84 & 22.33 & 39.37 & 26.01 & 6.80 & 1.65 & - & - & - & $\mathrm{ms}$ \\
\hline $100-150$ & 13.94 & 9.08 & 4.92 & tr. & - & 2.89 & 20.16 & 35.74 & 36.24 & 4.60 & 0.37 & - & - & - & fs \\
\hline
\end{tabular}




\section{Continue,}

\begin{tabular}{|c|c|c|c|c|c|c|c|c|c|c|c|c|c|c|c|}
\hline \multirow[b]{2}{*}{$\begin{array}{l}\text { Depth } \\
\text { (cm) }\end{array}$} & \multirow[b]{2}{*}{$\begin{array}{c}\text { EC } \\
\text { dS } \mathbf{~ m}^{-1}\end{array}$} & \multirow[b]{2}{*}{$\mathbf{p H}$} & \multirow{2}{*}{$\begin{array}{c}\mathrm{CaCO}_{3} \\
\%\end{array}$} & \multirow{2}{*}{$\begin{array}{c}\text { Gypsum } \\
\%\end{array}$} & \multirow[b]{2}{*}{$\begin{array}{c}\text { Gravel } \\
\%\end{array}$} & \multicolumn{9}{|c|}{ Particle Size Distribution } & \multirow[b]{2}{*}{$\begin{array}{r}\text { Texture } \\
\text { (USDA) }\end{array}$} \\
\hline & & & & & & $\begin{array}{c}1-2 \\
(\mathrm{~mm})\end{array}$ & $\begin{array}{l}1-0.5 \\
(\mathrm{~mm})\end{array}$ & $\begin{array}{c}0.5-0.25 \\
(\mathrm{~mm})\end{array}$ & $\begin{array}{c}0.25-0.125 \\
(\mathrm{~mm})\end{array}$ & $\begin{array}{c}\text { 0.125-0.063 } \\
(\mathrm{mm})\end{array}$ & $\begin{array}{c}<0.063 \\
(\mathrm{~mm}) \\
\end{array}$ & $\begin{array}{c}\text { Sand } \\
\%\end{array}$ & $\begin{array}{l}\text { Silt } \\
\%\end{array}$ & $\begin{array}{c}\text { Clay } \\
\%\end{array}$ & \\
\hline \multicolumn{16}{|c|}{ Soil Profile (13) } \\
\hline $0-15$ & 8.89 & 9.05 & 9.05 & - & - & 11.46 & 1.27 & 8.80 & 47.21 & 29.25 & 2.01 & - & - & - & fs \\
\hline $15-60$ & 34.49 & 8.56 & 1.67 & $\operatorname{tr}^{1}$ & 2.38 & - & - & - & - & - & - & 78.88 & 1.12 & 20.00 & $\mathrm{scl}$ \\
\hline $60-150$ & 13.54 & 8.02 & 0.00 & 7.06 & - & - & - & - & - & - & - & 79.50 & 0.28 & 20.22 & $\mathrm{scl}$ \\
\hline \multicolumn{16}{|c|}{ Soil Profile (14) } \\
\hline 0-30 & 9.00 & 7.97 & 3.76 & - & - & - & - & - & - & - & - & 79.98 & 0.01 & 20.01 & scl \\
\hline $30-70$ & 10.49 & 7.61 & 0.14 & 6.94 & 2.78 & - & - & - & - & - & - & 79.18 & 0.32 & 20.50 & $\mathrm{scl}$ \\
\hline $70-100$ & 11.33 & 7.78 & 0.00 & 6.97 & - & - & - & - & - & - & - & 78.50 & 1.00 & 20.50 & $\mathrm{scl}$ \\
\hline \multicolumn{16}{|c|}{ Soil Profile (15) } \\
\hline $0-15$ & 2.99 & 8.92 & 6.45 & - & - & 7.59 & 9.01 & 24.29 & 38.36 & 18.95 & 1.80 & - & - & - & fs \\
\hline $15-70$ & 25.51 & 8.54 & 2.60 & tr. & - & - & - & - & - & - & - & 77.99 & 2.01 & 20.00 & $\mathrm{scl}$ \\
\hline $70-150$ & 2.36 & 8.23 & 0.88 & 6.62 & - & - & - & - & - & - & - & 78.01 & 0.79 & 21.20 & $\mathrm{scl}$ \\
\hline \multicolumn{16}{|c|}{ Soil Profile (16) } \\
\hline 0-30 & 2.59 & 8.99 & 3.02 & - & - & 14.36 & 4.82 & 19.31 & 43.67 & 16.62 & 1.22 & - & - & - & fs \\
\hline $30-80$ & 29.80 & 7.67 & 0.74 & tr. & - & - & - & - & - & - & - & 79.15 & 0.80 & 20.05 & $\mathrm{scl}$ \\
\hline $80-150$ & 14.86 & 8.05 & 0.70 & 7.11 & - & - & - & - & - & - & - & 78.68 & 0.32 & 21.00 & $\mathrm{scl}$ \\
\hline \multicolumn{16}{|c|}{ Soil Profile (17) } \\
\hline $0-10$ & 4.22 & 8.98 & 7.01 & - & - & 17.87 & 4.41 & 16.83 & 38.61 & 20.33 & 1.95 & - & - & - & fs \\
\hline $10-30$ & 13.47 & 8.80 & 5.29 & $\operatorname{tr}^{1}$ & 2.50 & - & - & - & - & - & - & 78.20 & 1.80 & 20.00 & $\mathrm{scl}$ \\
\hline $30-80$ & 29.99 & 8.35 & 2.97 & 3.08 & - & - & - & - & - & - & - & 77.99 & 2.00 & 20.01 & $\mathrm{scl}$ \\
\hline 80-150 & 8.87 & 8.16 & 3.02 & 6.87 & - & - & - & - & - & - & - & 78.95 & 0.05 & 21.00 & $\mathrm{scl}$ \\
\hline \multicolumn{16}{|c|}{ Soil Profile (18) } \\
\hline 0-30 & 2.45 & 9.13 & 9.47 & - & 2.38 & 16.90 & 2.06 & 11.87 & 42.73 & 24.76 & 1.68 & - & - & - & fs \\
\hline $30-60$ & 17.65 & 8.52 & 0.74 & 7.21 & - & - & - & - & - & - & - & 78.11 & 1.39 & 20.50 & $\mathrm{scl}$ \\
\hline $60-100$ & 17.95 & 7.24 & 0.84 & 7.23 & - & - & - & - & - & - & - & 78.33 & 0.17 & 21.50 & $\mathrm{scl}$ \\
\hline
\end{tabular}

Abbreviations: ${ }^{1}$ : tr.-traces, ${ }^{2}$ : ls-loamy sand; scl-sandy clay loam; fs-fine sand; ms-medium sand; vfs-very fine sand 


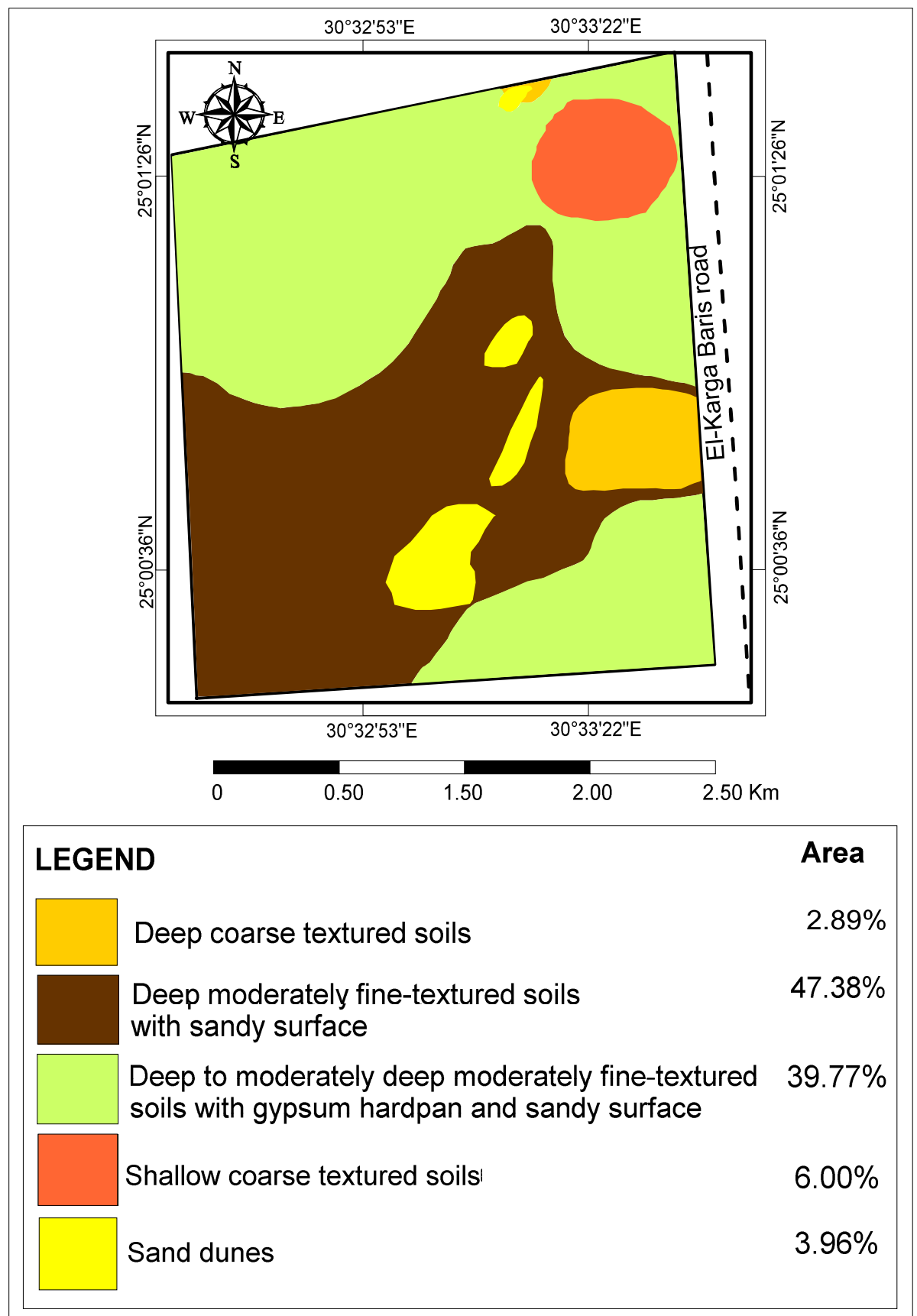

Fig. 4: Soil types and their spatial distribution within the investigated area

\section{Assessment of Landuse for Agriculture}

\subsection{Land Capability Classification}

Land capability is classified by USDA on the basis of lasting limitations or hazards in its use from the viewpoint of conserve the soil permanently productive. Consequently, soils of the studied area were evaluated according to their capability for agriculture based on six soil factors, which are: surface texture, soil depth, slope, erosion, permeability, and surface runoff, Table (4). Accordingly, the soils set in three classes as illustrated in Fig. (5). These capability classes are II, III, and VI represented 3.55, 86.58 , and $6 \%$ of the researched portion, respectively. Based on these results, there is $90.13 \%$ of the studied area is suitable for agriculture with moderate to special conservation practices. The class VI and sand dunes are excluded of cultivation where they can be used for other purpose. 
Table 4: Land Capability indices and classes of the studied soils

\begin{tabular}{|c|c|c|c|c|c|c|c|}
\hline $\begin{array}{l}\text { Profile } \\
\text { No. }\end{array}$ & $\begin{array}{l}\text { Surface } \\
\text { Texture }\end{array}$ & Depth & Slope $^{1}$ & Erosion $^{2}$ & Permeability $^{3}$ & $\begin{array}{l}\text { Surface } \\
\text { Runoff }^{4}\end{array}$ & $\begin{array}{c}\text { Capability } \\
\text { class }\end{array}$ \\
\hline 1 & Coarse & Shallow & NL & $\mathrm{N}$ & V.S & $\mathrm{S}$ & VI \\
\hline 2 & Coarse & Moderately Deep & NL & $\mathrm{N}$ & $\mathrm{S}$ & $\mathrm{S}$ & III \\
\hline 3 & Coarse & Moderately Deep & NL & $\mathrm{N}$ & $\mathrm{S}$ & $\mathrm{S}$ & III \\
\hline 4 & Coarse & Deep & NL & $\mathrm{N}$ & $\mathrm{S}$ & $\mathrm{S}$ & III \\
\hline 5 & Coarse & Deep & NL & $\mathrm{N}$ & $\mathrm{R}$ & $\mathrm{S}$ & III \\
\hline 6 & Coarse & Deep & NL & $\mathrm{N}$ & $\mathrm{S}$ & $\mathrm{S}$ & III \\
\hline 7 & Coarse & Deep & NL & $\mathrm{N}$ & $\mathrm{S}$ & $\mathrm{S}$ & III \\
\hline 8 & Coarse & Deep & NL & $\mathrm{N}$ & $\mathrm{S}$ & $\mathrm{S}$ & III \\
\hline 9 & Coarse & Deep & NL & $\mathrm{N}$ & $\mathrm{S}$ & $\mathrm{S}$ & III \\
\hline 10 & $\begin{array}{l}\text { Moderately } \\
\text { fine }\end{array}$ & Deep & NL & $\mathrm{N}$ & $\mathrm{S}$ & $\mathrm{S}$ & II \\
\hline 11 & Coarse & Deep & NL & $\mathrm{N}$ & $\mathrm{S}$ & $\mathrm{S}$ & III \\
\hline 12 & Coarse & Deep & NL & $\mathrm{N}$ & $\mathrm{R}$ & $\mathrm{S}$ & III \\
\hline 13 & Coarse & Deep & NL & $\mathrm{N}$ & S & S & III \\
\hline 14 & $\begin{array}{l}\text { Moderately } \\
\text { fine }\end{array}$ & Moderately Deep & NL & $\mathrm{N}$ & S & $\mathrm{S}$ & II \\
\hline 15 & Coarse & Deep & NL & $\mathrm{N}$ & $\mathrm{S}$ & $\mathrm{S}$ & III \\
\hline 16 & Coarse & Deep & NL & $\mathrm{N}$ & $\mathrm{S}$ & $\mathrm{S}$ & III \\
\hline 17 & Coarse & Deep & NL & $\mathrm{N}$ & $\mathrm{S}$ & $\mathrm{S}$ & III \\
\hline 18 & Coarse & Moderately Deep & NL & $\mathrm{N}$ & $\mathrm{S}$ & $\mathrm{S}$ & III \\
\hline
\end{tabular}

Abbreviations:

${ }^{1}$ : NL-nearly level ${ }^{2}$ : N-none ${ }^{3}$ : V.S- very slowly; S-slow; R-rapid ${ }^{4}$ : S-slow

\section{Soils with land capability class II}

This class included a part of SMU2 and SMU3 where their soils suited for cultivation over a long period of time. However, soils in this class have particular limitations like the contents of gypsum and salts that appear at levels that qualify them to a moderate degree of impact on productivity. Besides, the poorly permeability related to soils of profile 14 can lead to the rising of a groundwater level that may have a negative impact in the case of not rationalizing the irrigation process. These limitations require moderate ongoing management practices or slightly restrict the range of crops or both.

\section{Soils with land capability class III}

This class covered SMU1 and most of SMU2 and SMU3 in the studied area. These soils are good for cultivated crops, but have severe limitations such as increasing contents of salts and gypsum and/or limiting of effective depth by extremely hard layers. These restrictions can reduce the choice of crops and/or require special conservation practices that are more difficult to apply.

\section{Soils with land capability class VI}

This class involved of SMU4 which has unsuited soils for cultivation due to their severe limitations like shallowness, that restrict them for pasture or range, woodland, or wildlife food and cover. 


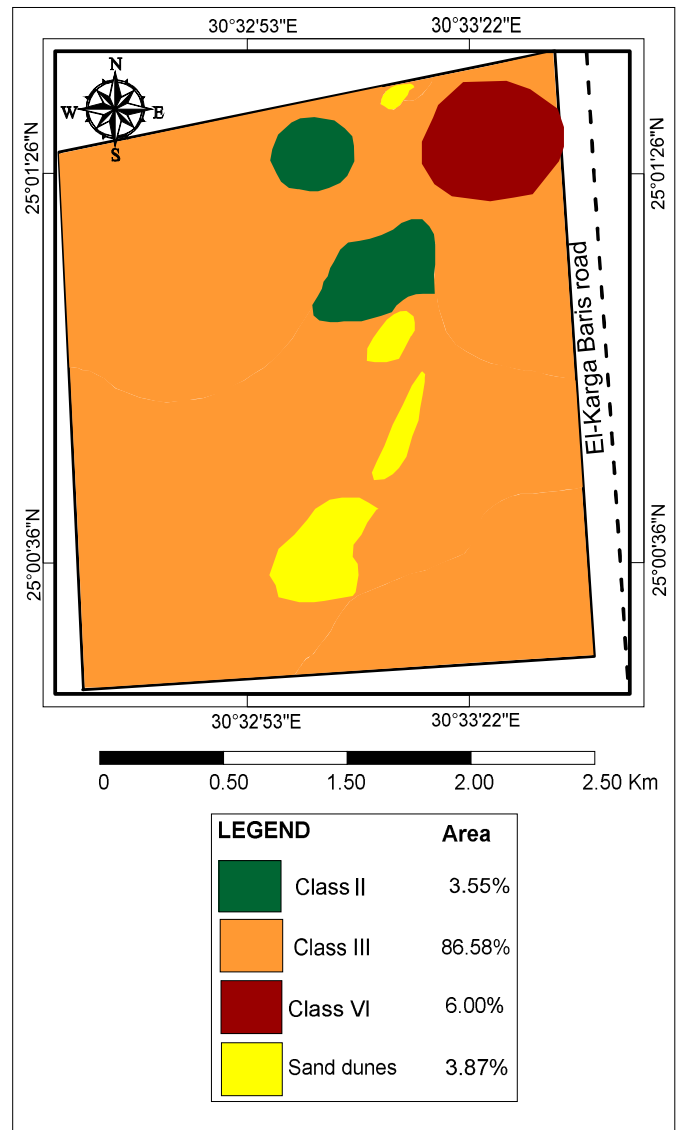

Fig. 5: Land capability classes of the studied area

Based on the previous feedbacks, it was found that the most important agricultural limitations in the studied portion are related to; (i) the effective depth of some soils that have an extremely hard layer at 90 to $130 \mathrm{~cm}$ depth, (ii) the poor permeability for some soils that have moderate fine texture, and (iii) high contents of salts and gypsum which have medium to high impact on productivity and cause a more limited choice of crops. Therefore, proposed soil management practices are suggested for reducing the effectiveness of agricultural limitations. These practices represented in establishing featured systems of subsurface drainage and irrigation in order to keep of land resource sustained for land use.

\section{Statistical analysis}

Descriptive statistics of weighted means concerning the estimated soil properties at the studied area showed that standard deviation ranged from $20.83-55.18$, while the mean values varied between 17.96 and 40.25. The coefficient of variation showed rats between 1.14-1.76. The statistical analysis illustrated that some of the studied soil profiles had homogeneity in their properties while the others were clearly varied. This is referring to the relative diversity of land resources within the investigated site, Fig. (6). 


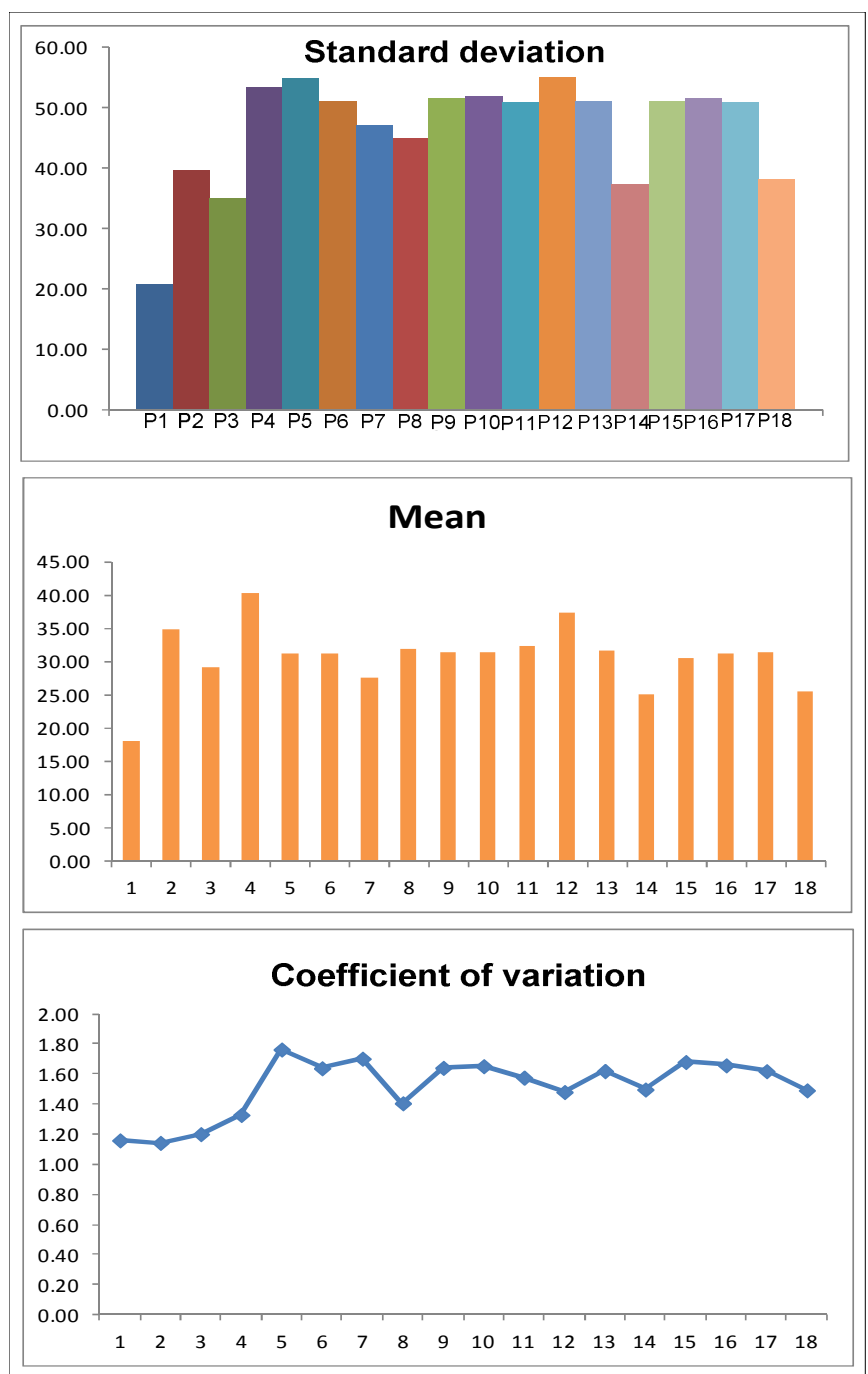

Fig. 6: Descriptive statistics of weighted means related to the estimated soil properties at the studied area

\section{Conclusions}

The specific goals of the article are; (i) to grasp of soil characteristics concerning to the study area as a model (1000 fed.), (ii) to characterize of soil types and their spatial distribution on basis of a semi-detailed grid sampling technique, and (iii) to assess of the investigated land resources for agriculture.

From the research that has been conducted, it is possible to conclude that the studied area could be distinguished into; (1) deep coarse textured soils which represented $2.89 \%$ of the investigated site, (2) deep moderately-fine textured soils with sandy surface and covered an area of $47.38 \%$, (3) deep to moderately deep moderately-fine textured soils with gypsum hardpan and sandy surface which comprised an area of $39.77 \%$, and (4) shallow coarse textured soils that included an area of $6 \%$.

These soils have been evaluated and hence classified according to their capability into classes II, III, and VI represented 3.55, 86.58, and $6 \%$ of the researched portion, respectively. Soils of class II suitable for cultivation however it requires moderate ongoing management practices. Soils belonging to class III are good to cultivated crops, but have severe limitations which need to special conservation practices that are more difficult to apply. Soils related to class VI are excluded of cultivation and can be used for pasture or range, woodland, or wildlife food and cover. Based on these feedbacks, there is $90.13 \%$ of the investigated portion is suitable for agriculture with moderate to special conservation practices.

The most important agricultural limitations in the studied portion are related to; (i) the effective depth of some soils that have an extremely hard layer at 90 to $130 \mathrm{~cm}$ depth, (ii) the poor permeability 
for some soils that have moderate fine texture, and (iii) high contents of salts and gypsum which have medium to high impact on productivity and cause a more limited choice of crops. All these limitations should be taken in consideration while planning for agricultural landuse within the investigated area.

\section{References}

Abu Seif, E.S., 2013. Performance of cement mortar made with fine aggregates of Dune Sand, Kharga Oasis, and Western Desert, Egypt: an experimental study. Jordan J. Civ. Eng., 7 (3):270-284.

Beadnell, H.J.L., 1933. Remarks on the prehistoric geography and underground waters of Kharga Oasis. Geogr. J., 81 (2):128-134.

Brady, N.C. and R. Weil, 2016. The Nature and Properties of Soils, 15th Edition, Pearson, 1104.

CONOCO, 1987. Geological map of Egypt (scale 1:500,000).

El-Sankary, M.M., 2002. Geological, sedimentological and radioactivity studies of the quaternary sediments, El Kharga Depression, Western Desert, Egypt (Ph.D. thesis), Ain Shams Univ., Cairo, Egypt, 241.

ESRI, 2006. In: “Arc-GIS User Manual”. Version 9.2. Redlands. California.

Gad, A., 2015. Land capability classification of some western desert Oases, Egypt, using remote sensing and GIS. The Egyptian Journal of Remote Sensing and Space Sciences, 18: S9-S18.

Gameh, M.A., N.M.K. Roshdy, M.A. Eissa, and M.M. Ahmed, 2017. Effect of Cultivation on the Soil Properties of El-Kharga Farm, New Valley, Egypt. Assiut J. Agric. Sci., 48 (1-1): 356-373.

Klingebiel, A.A. and P.H. Montgomery, 1961. Land Capability Classification. In: "Agriculture Handbook No. 210". USDA, Soil Conserv. Serv., Washington, DC.

Lagacherie, P., and A.B. McBratney, 2004. Spatial Soil Information Systems and Spatial Soil Inference Systems: perspectives for digital soil mapping. DSM Montpellier 13-17 September 2004. Elsevier, (Cited from Pasztor, L. and Szabo, J. 2006).

Omran, E.S.E., 2016. A simple model for rapid gypsum determination in arid soils. Model. Earth Syst. Environ., 2:185.

Page, A.L., R.H. Miller and D.R. Keeney, 1982. In: "Methods of Soil Analysis". Part 2. Chemical and Microbiological Properties. Agronomy, No. 9. Soil Sci. Soc. Am. Madison, WI 1159.

Rossiter, D.G., 2004. Digital soil resource inventories: status and prospects. Soil Use \& Management, 20(3): 296-301.

Salman, A.B., F.M. Howari, M.M. El-Sankary, A.M. Wali, and M.M. Saleh, 2010. Environmental impact and natural hazards on Kharga Oasis monumental sites, Western Desert of Egypt. Afr. Earth Sci., 58: 341-353.

Soil Survey Staff, 2017. In: 'Soil Survey Manual". Ditzler, C., K. Scheffe and H.C. Monger (eds.). USDA Handbook 18. Government Printing Office, Washington, D.C., USA, 603.

SPSS., 2014. SPSS Base 21.1 for Windows User's Guide. SPSS Inc., Chicago IL. 